Classification

Physics Abstracts

$64.70 \mathrm{~K}-81.30 \mathrm{H}$

\title{
Calculation of phase diagrams for the order-disorder transition in the binary systems $\mathrm{Ni}-\mathrm{Fe}$ and $\mathrm{Ni}-\mathrm{Al}$
}

\author{
P. Cenedese, A. Marty and Y. Calvayrac \\ Centre d'Etudes de Chimie Métallurgique, C.N.R.S. LP 2801, 15 rue Georges Urbain, \\ 94407 Vitry sur Seine Cedex, France
}

(Reçu le 31 janvier 1989, accepté sous forme définitive le 5 mai 1989)

\begin{abstract}
Résumé. - La Méthode de Variation des Amas, dans l'approximation du Tétraèdre-Octaèdre, est utilisée pour étudier le diagramme d'équilibre des phases au voisinage de la transition ordredésordre, pour $\mathrm{Ni}_{3} \mathrm{Al}$ et $\mathrm{Ni}_{3} \mathrm{Fe}$. Les potentiels d'interaction de paires sont déterminés à partir de mesures de diffusion diffuse des rayons $\mathrm{X}$ ou des neutrons. Les diagrammes de phase calculés sont comparés aux données expérimentales. On montre que, dans le cas de $\mathrm{Ni}_{3} \mathrm{Fe}$, un accord satisfaisant peut être obtenu en introduisant une faible variation du potentiel d'interaction de paires de seconds voisins en fonction de la concentration.
\end{abstract}

Abstract. - The Cluster Variation Method, in the Tetrahedron-Octahedron approximation, is used to investigate the $\mathrm{Ni}_{3} \mathrm{Al}$ and $\mathrm{Ni}_{3} \mathrm{Fe}$ ordering phase diagrams. The pairwise interaction potentials used are extracted from X-ray or neutron diffuse scattering measurements. The calculated phase diagrams are compared to the experimental data. It is shown that, for the case of $\mathrm{Ni}_{3} \mathrm{Fe}$, a satisfactory agreement can be achieved by introducing a slight variation of the secondneighbor pair interaction potential as a function of concentration.

\section{Introduction.}

The degree of order plays an important role in the physical properties of alloys, and it is therefore important to know its variation as a function of temperature and concentration. In an Ising type model, the calculation of phase diagrams, near the order-disorder transition, requires the knowledge of the pair potentials $V_{i}=\left(V_{i}^{\mathrm{AA}}+V_{i}^{\mathrm{BB}}-2 V_{i}^{\mathrm{AB}}\right) / 4$ where $V_{i}^{\mathrm{AB}}$ is the pair interaction potential between $i$-th neighbors A and B atoms. Several classes of approach are possible :

- the pair interaction potentials may be theoretically derived ab initio from the electronic structure of the alloy by using approximated techniques like the pseudo-potential method. The phase diagram may then be calculated using the Cluster Variation Method (CVM) or the Monte Carlo method (MC). However, the electronic energy calculations are still not sufficiently accurate to derive the pair interaction potentials from first principles, although some important steps have recently been made in this direction $[1,2]$;

- another class of approach consists in using phenomenological models with a minimum number of parameters that are chosen in order to best fit the experimental thermodynamical 
data. The interaction energies are obtained from the comparison of calculated and observed phase transition temperatures. In fact, this amounts to a kind of fitting between calculated and observed equilibrium diagrams ;

- a third approach consists in using an independent way to determine the interaction energies from which phase diagrams may be calculated and compared with those experimentally observed. This is the way we have followed in the present work. The energetic parameters are obtained from X-ray and neutron diffuse scattering measurements, using the Inverse Cluster Variation Method (ICVM), for the $\mathrm{Ni}$ rich $\mathrm{Ni}-\mathrm{Al}$ and $\mathrm{Ni}-\mathrm{Fe}$ systems. Phase diagram portions are calculated using the CVM and the MC methods. The relevancy of the pair interaction potentials is revealed through the comparison of these phase diagrams with those experimentally observed.

\section{Pair potential determination.}

The degree of short range order of a binary solid solution, which depends on the reduced energies $V_{i} / k_{\mathrm{B}} T$, can be accurately determined by standard analysis of diffuse scattering experiments. Within the CVM formalism the free energy functional $\beta F=\beta E-S / k_{\mathrm{B}}$ of the Ising model can be written as

$$
\beta F=\Sigma_{i} \mu_{i} \beta V_{i} X_{i}+\Sigma_{i} a_{i} \mu_{i} \operatorname{Tr}^{(k)} \gamma_{i}^{k} \rho_{i}^{k} \ln \rho_{i}^{k}
$$

where

$$
\beta=1 / k_{\mathrm{B}} T \text { and } X_{i}=4 c_{\mathrm{A}} c_{\mathrm{B}} \alpha_{i}+\left(c_{\mathrm{A}}-c_{\mathrm{B}}\right)^{2}
$$

in which we have distinguished

- the internal energy $\beta E$, where appear the unknown parameters $\beta V_{i}$ conjugated to the experimentally determined pair correlation functions $X_{i}$ with multiplicity $\mu_{i}$. The $X_{i}$ are given in (1) as functions of the concentration and the Warren-Cowley short range order parameters $\alpha_{i}$;

- the entropy $S$, which is calculated in CVM by approximating the total density matrix by a product of a finite number of type of cluster density matrices [3]. So, in the above expression of $S, a_{i}$ stands for the CVM coefficient of the cluster of type $i$ associated with probabilities $\rho_{i}^{k}$ each of degeneracy $\gamma_{i}^{k}$.

Once the basic cluster is specified, and if the pair interactions are fixed, the equilibrium values of the restricted set of correlation functions are determined by minimizing the free energy. Now, if instead of fixing the pair potentials we fix the pair correlation functions, we note that in (1) the pair interactions behave as Lagrangian multipliers. Thus their determination proceeds from the constrained minimization of $\beta F$. We have used as basic cluster a layer shaped quadruple tetrahedron (QT) [4]. This cluster allows one to accurately compute the first four pair interactions in the FCC lattice as long as $V_{1}$ is large compared to the other potentials.

\section{Phase diagrams.}

3.1 Ni-Al SYSTEM. - First we have chosen to study the Ni-rich $\mathrm{Ni}-\mathrm{Al}$ system for two main reasons. i) Carefully made experimental and theoretical phase diagrams are available and some discrepancy exits between the results published by different authors [5]. ii) Short range order (SRO) parameters have been determined from X-ray diffuse scattering experiments, and effective pair interaction potentials can be calculated from these. 
SRO parameters for $\mathrm{Ni}-10$ at $\% \mathrm{Al}$ have been recently reported [6, 7]. We have determined pair potentials from the SRO parameters $\alpha_{i}$ given in these two papers. The results strongly differ, for an alloy of nearly the same composition. From the data of reference [7] the sign of $V_{2}$ is negative and longer range interactions (up to $V_{4}$ ) must be considered for the system to order in the $\mathrm{L}_{2}$ structure. The measurements reported in [7] are performed on samples quenched from $973 \mathrm{~K}$. Hence it may be suspected that the samples have states of order corresponding to frozen dynamic states and that the $V_{i} / V_{1}$ ratios are thus affected. Moreover since the measurements are performed at room temperature, thus the contribution from thermal diffuse scattering is important and may be overestimated. Accordingly we have not used the data obtained from [7]. The pair potentials we have determined from [6] are listed in table I, together with the $\alpha_{i}$ values from which they have been obtained. These SRO parameters have been measured at $120 \mathrm{~K}$ on a single crystal quenched from $673 \mathrm{~K}$.

Table I. - SRO parameters $\alpha_{i}$ and pair interaction potentials $V_{i}$ (in meV) for a Ni-10.5 at \% Al alloy. The values obtained for the pair interaction potentials correspond to the $\mathrm{L1}_{2}$ ground state in Kanamori's stability map [8].

$\begin{array}{ccccc}i & 1 & 2 & 3 & 4 \\ & & & & \\ \alpha_{i} & -0.101 & 0.110 & 0.002 & 0.071 \\ V_{i} & 34.4 & -4.2 & -1.4 & -6.1\end{array}$

In the $\mathrm{Ni}-\mathrm{Al}$ system, it is impossible to quench a single phase supersaturated solid solution when the atomic percentage of aluminium is larger than $10.5 \%$. Thus, to compute the coherent $\mathrm{Ni}$-rich $\mathrm{Ni}$-Al phase diagram part we have assumed that the pair potentials do not explicitly depend on the concentration. The calculations were carried out both in CVM and MC.

- The CVM computational effort when dealing with ordered phases depends strongly both on the size and on the shape of the chosen basic clusters which in turn depend on the range of the interactions. In general, due to the symmetry breakdown at the transition, the basic clusters of the disordered phase split in several clusters of lesser symmetry making a CVM treatment tedious and difficult. Thus we are concerned by introducing some approximations which partially avoid these difficulties.

The basis of the approximations considered is that at low temperatures the major contribution to the free energy comes from the internal energy so that we do not need very refined approximations for the entropy. So we consider approximations which can satisfactorily handle long range interactions without complicating the entropy expression. In the context of the $\mathrm{Ni}$-Al system, the simplest method consists in choosing simple basic clusters such as the Tetrahedron $(\mathrm{T})$ and Octahedron $(\mathrm{O})$ to describe the entropy of the system, and to include the third and fourth neighbor interactions in the internal energy through the BraggWilliams approximation. In this « hybrid " CVM, only 29 correlation functions are needed to characterize the $\mathrm{L}_{2}$ structure, whilst in comparison 82 are needed in the QT when describing the simple FCC phase. The phase diagram we obtain is shown in figure 1 (dashed lines).

- To check the incidence of our approximation on the phase diagram topology, grand canonical MC calculations have been performed using the Metropolis importance sampling. The grounds of our MC calculations are the following: for given energy parameters $V_{i}$ and relative chemical potential $\mu / V_{1}$, the concentration (order parameters...) are direct results of MC simulations, thus it is possible to obtain the temperature versus concentration 


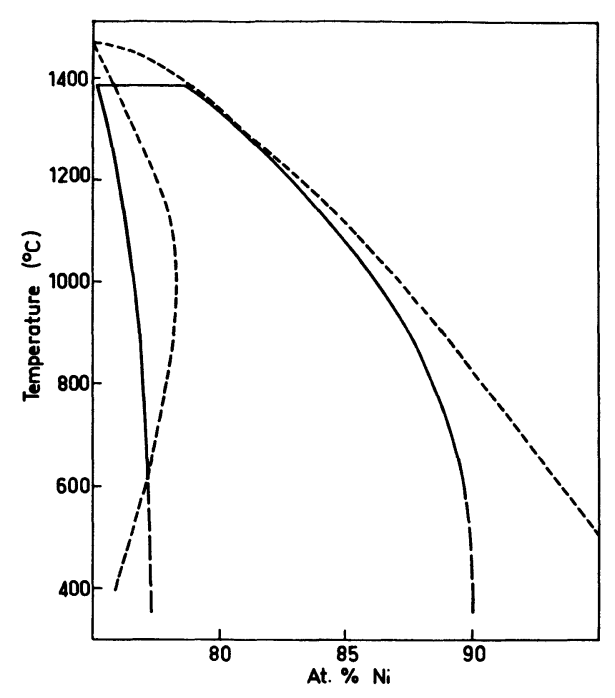

Fig. 1.

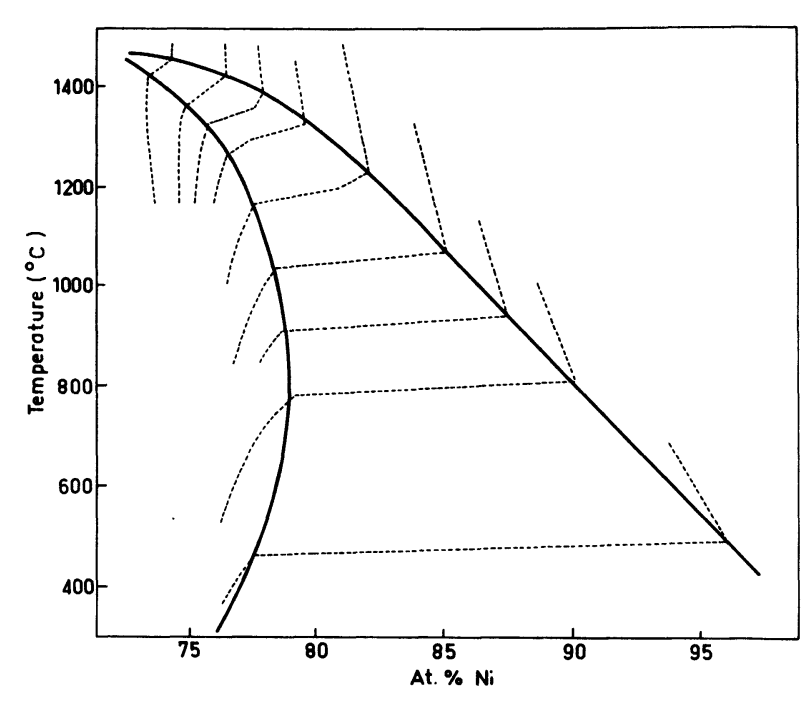

Fig. 2.

Fig. 1. - Part of the Ni-Al phase diagram : full lines from Hansen's handbook, dashed lines from this work, calculated in the (TO + BW)-CVM approximation.

Fig. 2. - Part of the Ni-Al phase diagram, calculated by Monte Carlo simulation.

curve, which is a smooth function as long as the computations are done in a single phase domain. In general this curve is roughly a straight line [9] except for the following cases :

- at very low temperature, where it behaves singularly, the concentration at zero $\mathrm{K}$ being a stepwise function of the chemical potential ;

- at some particular temperature where a transition between two phases occurs. Thus, in the MC method starting from the $\mathrm{L1}_{2}$ ordered phase, by plotting the temperature $T$ versus the concentration $C_{\mathrm{A}}\left(V_{i}, T, \mu\right)$, for a given set of energy parameters $V_{i}$, at a constant relative chemical potential $\mu$ (dotted lines in Fig. 2), $T$ remains constant in a concentration range (horizontal segments) which corresponds to a two-phase region of the phase diagram. The extremities of these horizontal segments give us the phase diagram (Fig. 2).

It is obvious in figure 2 that some segments are not strictly horizontal. This is particularly sensitive when the chemical potential value is close to the value where a congruent transition occurs, that is when the two phase domain shrinks. This is mainly due to finite size effects in our MC calculations where only FCC boxes of $4 \times 8^{3}$ and $4 \times 10^{3}$ sites were considered, and to a temperature scanning grid too large.

The agreement between MC and CVM phase diagrams is excellent, showing that the chosen CVM approximation is good enough to treat ordered phases even at low temperature. A slight difference can be seen near the congruent point, which may be attributed to the small size of the sample in the MC simulation (2 048 sites). The topology of the diagram is similar to the one obtained by Mohri et al. [10] for a $V_{2} / V_{1}$ ratio $=-0.2$, and is rather insensitive to $V_{3}$ and $V_{4}$.

The $\mathrm{A} 1-\mathrm{L} 1_{2}$ two phase boundary can also be compared with the experimental phase diagram from Hansen's handbook (continuous lines in Fig. 1). At high temperature the overall agreement with experiment is reasonable. The value of the virtual transition temperature at the metastable congruent point $(1744 \mathrm{~K})$ is in very good agreement with the 
value of $1723 \mathrm{~K}$ estimated by Cahn et al. [11] from experimental results. However, the overall agreement with experiment worsens at low temperatures, even if we take into account the fact that for low aluminium contents the experimental phase diagram is still controversial [5].

Finally, we note that in our phase diagram calculation, we start from an experimental equilibrium SRO state belonging to the A1 phase and, under the same conditions of temperature and concentration, we end at a state belonging to the two phase domain. We think that the assumption of concentration independent pair potentials leads to this lack of self-consistency.

3.2 Ni-Fe SYSTEM. - The phase diagram of the $\mathrm{Ni}_{3} \mathrm{Fe}$ system has a very simple shape. There is a first order $\mathrm{L1}_{2}-\mathrm{A} 1$ transition around the stoichiometry. The boundaries of the two-phase region between the ordered and the disordered phase have been carefully determined using Mössbauer spectroscopy [12] and thus a comparison with theoretical calculations can be done. Short range ordered states in this alloy have been carefully studied by Lefebvre et al. [13, 14] using thermal neutron scattering experiments. From the results obtained by these authors on a single crystal quenched from 780 and $808 \mathrm{~K}$, we have estimated pair interaction potentials. The values obtained are listed in table II, together with the $\alpha_{i}$ values taken from $[13,14]$.

Table II. - Pair interaction potentials $V_{i}$ (in meV) for a Ni-23.5 at $\% \mathrm{Fe}$ alloy, obtained from two sets of short range order parameters $\alpha_{i}$ measured after quenching from 780 and $808 \mathrm{~K}$.

$\begin{array}{ccccc}i & 1 & 2 & 3 & 4 \\ \alpha_{i}(780 \mathrm{~K}) & -0.120 & 0.151 & -0.013 & 0.058 \\ V_{i}(780 \mathrm{~K}) & 16.86 & -5.13 & 1.01 & 0.21 \\ \alpha_{i}(808 \mathrm{~K}) & -0.110 & 0.138 & -0.010 & 0.055 \\ V_{i}(808 \mathrm{~K}) & 15.03 & -5.50 & 0.55 & -0.65\end{array}$

It should be noted that, in the case of $\mathrm{Ni}_{3} \mathrm{Fe}$, equilibrium short range ordered states may be expected to be correctly frozen by quenching from these temperatures. Hence the $V_{i}$ values can be used with confidence to estimate physical data and compare the values obtained to available measurements. Thus we give in figure 3 the dependence of the equilibrium long range order parameter $\eta$ with temperature, calculated from the $V_{i}$ obtained at $808 \mathrm{~K}$, for the stoechiometric $\mathrm{Ni}_{3} \mathrm{Fe}$ alloy. We note an excellent agreement with the value at $723 \mathrm{~K}$ accurately measured from the integrated intensities of all the superstructure lines of a

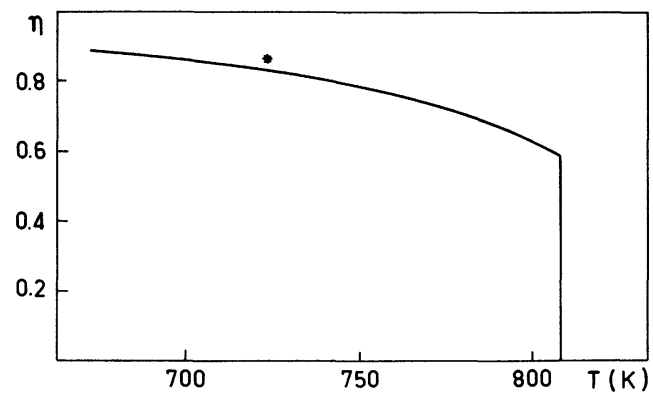

Fig. 3. - Dependence of the long range order parameter $\eta$ on the temperature, for a $\mathrm{Ni}_{3} \mathrm{Fe}$ alloy, calculated in the (TO + BW)-CVM approximation; $(*)$ experimental value from reference [15]. 
$\mathrm{Ni}_{3} \mathrm{Fe}$ powder sample [15]. This plot shows that a first order phase transition occurs at $808 \mathrm{~K}$. This temperature is $23 \mathrm{~K}$ higher than the observed disordering temperature (785 K). Therefore the transition temperature so calculated is in very good agreement (with the accuracy of $2.9 \%$ ) with the corresponding experimentally observed temperature.

If we compare the values of the pair interaction energies at 780 and $808 \mathrm{~K}$ (Tab. II), we see that, although $V_{3}$ and $V_{4}$ are small, they vary noticeably with temperature. As shown by Gautier [16], the interchange energies in a ferromagnetic alloy depend on the temperature through the magnetic state of order. However, far from the Curie temperature and within a temperature range of a few tenth of degrees, it seems difficult to attribute such a change of the $V_{3}$ and $V_{4}$ values to magnetic effects only. So we consider that only the first two potentials are significant. As the variations of $V_{1}$ and $V_{2}$ with respect to temperature are small, in the following we will neglect the temperature dependence of these interaction energies.

Now, if the values of $V_{3}$ and $V_{4}$ are small and may be inaccurately evaluated, can they be omitted ? Using the set of pair interaction potentials obtained at $780 \mathrm{~K}$ (instead of the ones obtained at $808 \mathrm{~K}$ ), the calculated transition temperature is lowered by $50 \mathrm{~K}$. Reducing then the values of $V_{3}$ and $V_{4}$ to zero, the transition temperature is lowered by $80 \mathrm{~K}$. Hence, it appears that the value of the transition temperature is very sensitive to the long range potentials even if these are small with respect to $V_{1}$ and $V_{2}$. If we consider only $V_{1}$ and $V_{2}$, the two sets of pair potentials lead to transition temperatures differing by $20 \mathrm{~K}$ (or $3 \%$ ), while $V_{1}$ and $V_{2}$ change by 11 and $7 \%$ respectively. Such differences are within the expected experimental incertitude.

In view of these results we decided to consider only $V_{1}$ and $V_{2}$ in our phase diagram computation and to normalize the calculated curve to the experimental value at the congruent point. In the case of the $\mathrm{Ni}-\mathrm{Al}$ system, we concluded that a concentration dependence of the interaction energies must improve the agreement with the experimental phase diagram. For the $\mathrm{Ni}_{3} \mathrm{Fe}$ system the assumption of composition independent pair potentials leads to a CVM (TO [10] as TO $+\mathrm{BW}$ if $V_{3}$ and $V_{4}$ are added) phase diagram with undesirable features : the transition temperature increases continuously with increasing iron concentration, in contrast to the experimental phase diagram, where a clear maximum is found around the 73-27 composition.

The CVM approximation is particularly designed to deal with concentration dependent pair interactions, as the concentration is related to some correlation function in (1). At least two methods can be used :

- either the correlation function associated with the concentration is kept fixed during the minimization of the free energy, then the corresponding chemical potential is estimated from a simple thermodynamical derivative ;

- either the concentration constraint is taken into account by introducing a Lagrangian multiplier which at the solution turns to be the chemical potential.

The two methods give the same results and have been used to verify the coherency of the formalism. The phase diagram is computed by forming the grand potential for the two phases, and by comparing their values as well as the values of the chemical potentials instead of using the free energy common tangent method. It is to be noted that introducing concentration dependent interactions would lead to severe difficulties to estimate the phase boundaries from MC calculations.

In the absence of experimental SRO data for other compositions, we introduced a volume dependence through a phenomenological model where $V_{1}$ and $V_{2}$ are given as a first order Taylor expansion. It must be stressed that this procedure has no more physical significance than that it gives the concentration dependence of $V_{2} / V_{1}$ which appears to be necessary in order to obtain a realistic phase diagram. In this model an increase of $V_{2} / V_{1}$ of $12 \%$ when the 
iron concentration varies from 20 to 30 at $\%$, with a corresponding change on $V_{1}$ of about $1 \%$, have been found to be adequate.

The phase diagram so obtained is plotted in figure 4 together with the experimentally observed one [12]. The calculated curve has been normalized to the experimental value of the transition temperature at the 73-27 composition. The topology of the diagram agrees well with that experimentally determined : it may be noted particularly that, theoretically as experimentally, the maximum in transition temperatures is situated at a non-stoechiometric composition (near the 73-27 composition). At the stoechiometric composition a two-phase region is clearly seen, the extent of which is very similar in the calculated diagram and in the experimentally observed one.

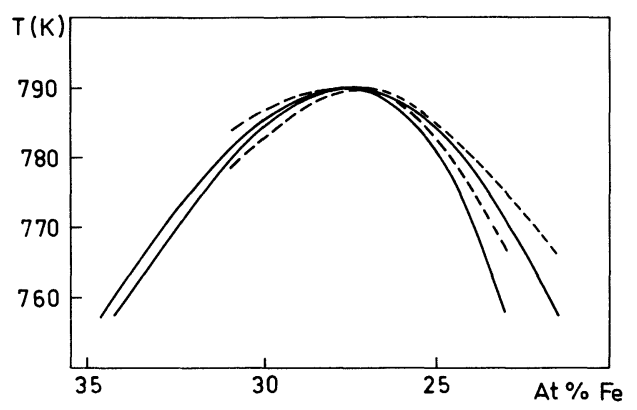

Fig. 4. - Phase diagram for the order-disorder transition near $\mathrm{Ni}_{3} \mathrm{Fe}$. Full lines : calculated in the TOCVM approximation, with the assumption of concentration dependent pair interaction potentials. Dashed lines : experimentally observed diagram, from reference [12].

\section{Conclusion.}

A theoretical investigation of the $\mathrm{Ni}_{3} \mathrm{Al}$ and $\mathrm{Ni}_{3} \mathrm{Fe}$ ordering phase diagrams was made using the Tetrahedron-Octahedron approximation of the CVM, modified in order to take into account the third and fourth-neighbor pair interaction energies through the Bragg-Williams approximation. The values of the interaction energies used have been determined in an independent way, from X-ray or neutron diffuse scattering experiments. The following results have been obtained :

- the incidence of the approximation on the phase diagram topology was tested on the NiAl system by comparing with the phase boundaries deduced from Monte-Carlo simulations. The agreement is excellent;

- for the $\mathrm{Ni}-\mathrm{Al}$ system, the topology of the calculated diagram is similar to the one obtained by Mohri et al. using the T-O approximation of the CVM: no effect of $V_{3}$ and $V_{4}$ has been detected;

- for the $\mathrm{Ni}-\mathrm{Al}$ system, the agreement between the calculated diagram and the experimental one is reasonable at high temperature. However the agreement with experiment worsens at low temperature. This behavior is thought to be due to the use of concentration independent potentials ;

- for the $\mathrm{Ni}-\mathrm{Fe}$ system, the values of $V_{3}$ and $V_{4}$ are small with respect to $V_{1}$ and $V_{2}$ and seems to have been inaccurately determined. However, the calculated values of the transition temperatures are rather sensitive to $V_{3}$ and $V_{4}$. A good agreement with the experimental value at the stoichiometry is obtained if the first four potentials are taken into account ; 
- a maximum near the $\mathrm{Ni}_{3} \mathrm{Fe}$ stoichiometry cannot be obtained in the approximation we used, unless concentration dependent pair interactions are considered;

- such a concentration dependence was introduced through a simple phenomenological expression. A realistic phase diagram was obtained for a $V_{2} / V_{1}$ ratio which increases by $12 \%$ when the iron concentration varies from 20 to 30 at \%. Further experimental investigation of the concentration dependence of the pair interactions in this system would be very interesting in support of this result, although this would require an accuracy in the measurements which seems difficult to achieve. Theoretical calculations of $V_{i}$ as a function of concentration (from electron theory) would be very helpful, the available «experimental » interaction energies being used as reference data.

Anyway this study shows that by using an improved CVM approximation together with accurately determined « experimental » interaction energies, plausible phase diagrams can be predicted. The method is promising for ternary systems where much information on the phase diagrams is lacking.

\section{References}

[1] Bieber A., Gautier F., Treglia G., Ducastelle F., Solid State Commun. 39 (1981) 149.

[2] Bieber A., Gautier F., Acta Metall. 34 (1986) 2291.

[3] Sanchez J. M., Ducastelle F., Gratias D., Physica 128A (1984) 334.

[4] Finel A., Thèse, Université Pierre et Marie Curie, Paris (1986).

[5] Sigli C., Sanchez J. M., Acta Metall. 33 (1985) 1097.

[6] Chassagne F., Thèse, Université Pierre et Marie Curie, Paris (1986);

also : Chassagne F., Bessiere M., Calvayrac Y., Cenedese P., Lefebvre S., Acta Metall. (in press).

[7] Klaiber F., Schonfeld B., Kostorz G., Acta Crystallogr. A43 (1987) 525.

[8] Kanamori J., Kakehashi Y., J. Phys. 12 (1977) C7-274.

[9] Kikuchi R., Cahn J. W., Acta Metall. 27 (1979) 1337.

[10] Mohri T., Sanchez J. M., De Fontaine D., Acta Metall. 33 (1985) 1171.

[11] Cahn R. W., Siemers P. A., Geiger J. E., Bardhan P., Acta Metall. 35 (1987) 2737.

[12] Van Deen J. K., Van Der Woude F., Acta Metall. 29 (1981) 1255.

[13] Lefebvre S., Bley F., Bessiere M., Fayard M., Roth M., Cohen J. B., Acta Crystallogr. A 36 (1980) 1.

[14] Lefebvre S., Bley F., Fayard M., Roth M., Acta Metall. 29 (1981) 749.

[15] Calvayrac Y., Fayard M., C. R. Acad. Sc. Paris 275 (1972) C-1.

[16] Gautier F., High Temperature Alloys: Theory and Design, Ed. P. O. Stiegler (1984). 